\title{
A rare cutaneous lesion in the neonatal period: The non-Langerhans cell histiocytosis
}

\author{
Adnan BARUTCU $^{1}$ (D), Ferda OZLU ${ }^{2}$ (D), Hacer YAPICIOGLU YILDIZDAS ${ }^{2}$ (D), Mehmet SATAR ${ }^{2}$ (D) \\ ${ }^{1}$ Pediatric Clinic, Halfeti State Hospital, Sanliurfa, Turkey. \\ ${ }^{2}$ Divison of Neonatology, Department of Pediatrics, School of Medicine, Cukurova University, Adana, Turkey.
}

Corresponding Author: Adnan BARUTCU

E-mail: adnan_barutcu@hotmail.com

Submitted: 23.03.2020 Accepted: 07.06.2020

\section{ABSTRACT}

The non-Langerhans cell histiocytosis (non-LCH) is a group of diseases characterized by cutaneous involvement in the neonatal period. The non-LCH affects less than 1 in 200,000 children born each year. A definitive diagnosis is important for the treatment of the disease. Therefore, skin biopsies should be performed in neonates with cutaneous lesions as early as possible. Only in the presence of cutaneous involvement without systemic involvement, it does not require any treatment, because the lesions can mostly self-heal. Here, we present a neonate diagnosed with non-LCH following a skin biopsy. Keywords: Histiocytic disorders, Neonatal, non-Langerhans cell histiocytosis

\section{INTRODUCTION}

The histiocytic syndromes consist of a group of disorders that share in common the proliferation of cells of the monocyte/macrophage lineage. It has been conventional to divide the histiocytoses into two separate groups: Langerhans cell histiocytosis (LCH) and non-LCH. Histiocytosis affects 1 in 200,000 children born each year; non-LCH is much more rare [1]. The non-LCH are a group of disorders defined by the accumulation of histiocytes that do not meet the phenotypic criteria for the diagnosis of Langerhans cells.

\section{CASE REPORT}

A 2-day-old female neonate, born at 39 weeks of gestation with normal spontaneous vaginal delivery was referred to our hospital due to skin lesions. She was born with pinkish - red, palpable lesions with 5-15 $\mathrm{mm}$ in diameter, scattered all over the body including scalp (Figures 1,2). She was being breastfed by her mother. On physical examination, her vital signs were normal except palpable skin lesions. Laboratory tests, including complete blood count, peripheral smear, urinalysis, liver and kidney function tests, coagulation tests, procalcitonin, viral serology were all normal. Toxoplasmosis, other (including syphilis), rubella,cytomegalovirus, herpes simplex virus (TORCH) and total immunoglobulin (IgM) tests were all negative. Transabdominal and transcranial ultrasonographies were normal. Skin biopsy was performed and CD68 and S100 markers were found to be positive. As a result, a diagnosis of non-LCH was made. Birbeck granules were absent in the cells (Figures 3,4). In the follow-up of the patient, the lesions disappeared at the age of 6 months. At 10 months of age she was with no evidence of relapse. She is being followed-up at the Neonatology and Dermatology outpatient clinics. Written informed consent was obtained from the parents for publication of this case report including the photographs.

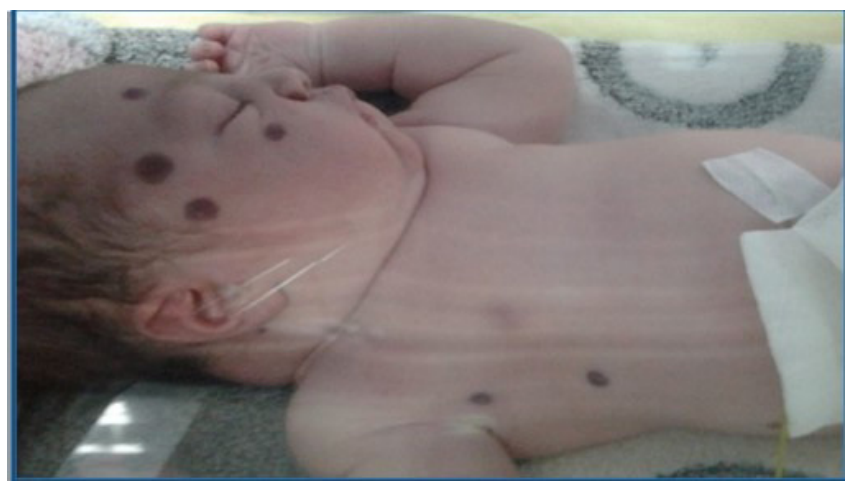

Figure 1. Palpable skin lesions

How to cite this article: Barutcu A, Ozlu F, Yapicioglu Yildizdas H, Satar M. A rare cutaneous lesion in the neonatal period: A non-Langerhans cell histiocytosis. Marmara Med J 2020;33: 160-162. doi: 10.5472/marumj.xx 


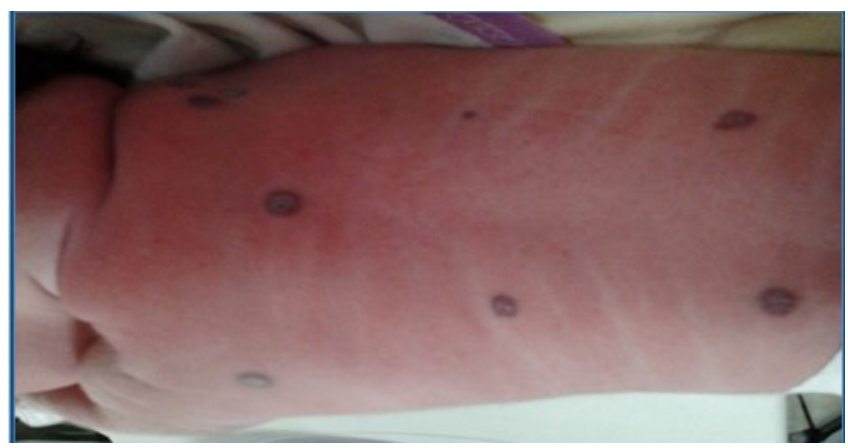

Figure 2. Palpable skin lesions

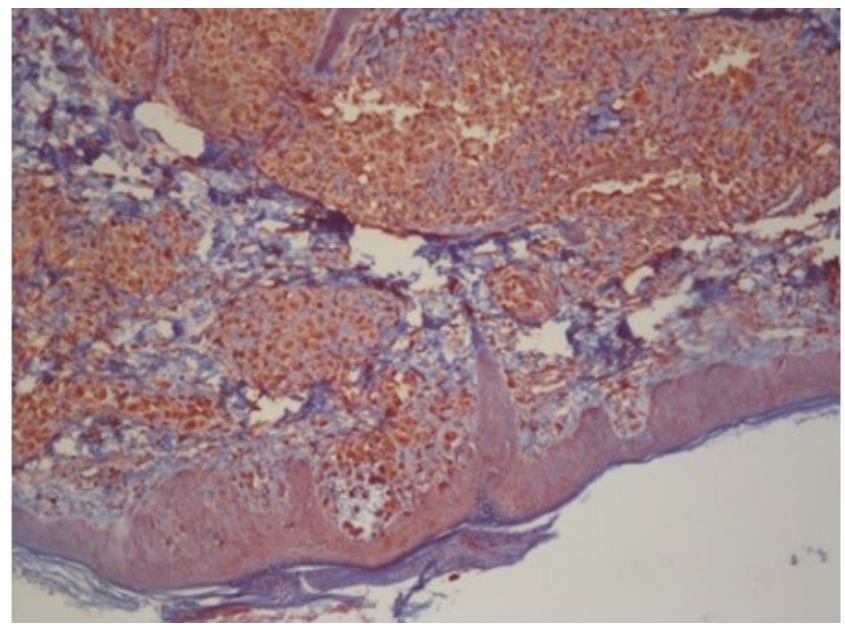

Figure 3. CD68 staining confirmed the existence of histiocytes

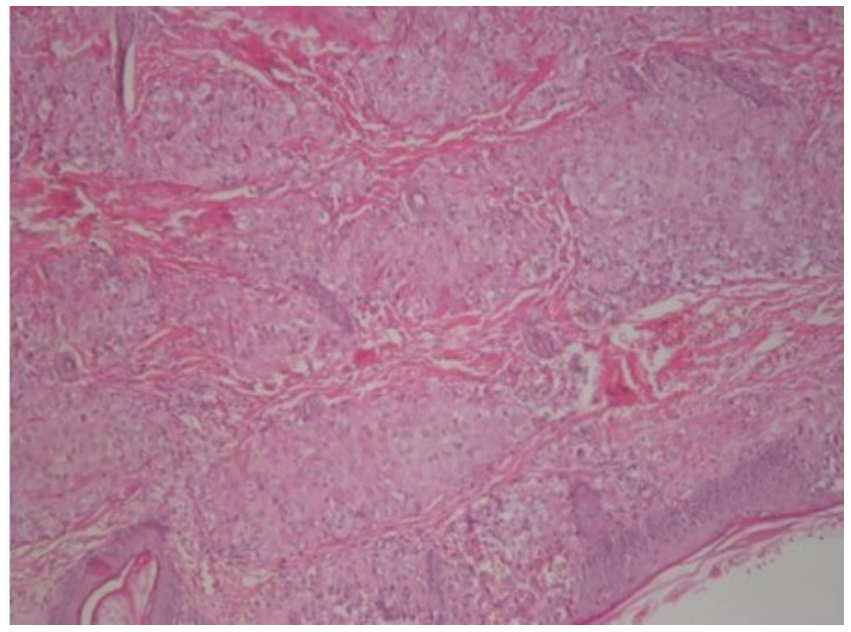

Figure 4. Sections of the lesion with histiocytosis (H and E x 100)

\section{DISCUSSION}

The non-LCH is a group of diseases with cutaneous involvement in the neonatal period [1,2]. Dermal dendrocyte infiltration is seen in this group of diseases, but typical Langerhans cells are absent. As reported by Classen, et al., some subtypes of these disorders typically occur early in life and tend to resolve spontaneously [3]. Our patient, also had multiple palpable lesions all over her body, with no systemic involvement. The lesions spontaneously disappeared by 6 month of age.

Clinically, the non-LCH can be divided into 3 groups. The first group that predominantly affects the skin; the second group that affects the skin but has a major systemic component such as hepatic, skeletal, central nervous system or lung involvement; and the third group that primarily involves extra-cutaneous sites, although skin may be also involved [4]. Some subtypes may be disseminated and life-threatening [5]. In childhood, only cutaneous involvement form limits itself $[6,7]$. In our patient, non-LCH affected skin predominantly.

Prompt evaluation of disease extent upon diagnosis is mandatory for risk-adapted treatment. Although, the prognosis cannot be predicted after the diagnosis, the course of the disease varies from spontaneous recovery to chronic course or fulminant deterioration [8].

Lesions which occur in the first 6 months of life can be either solitary or multiple. Lesions are about 5-20 $\mathrm{mm}$ in diameter, pinkish-red in color, firm, solid and limited well. In the follow-up, lesions can fade or they can heal with atrophic scars or hyperpigmentation in months or years. Diagnosis is made histopathologically [3].

The etiology and pathogenesis of non-LCH and its various clinical forms remain obscure. Viral, immunologic, and neoplastic mechanisms have been proposed, but none of them have been conclusively proven. Lesions originate from histiocytes and monocyte/macrophage derived giant cells. Macrophage surface markers of these patients are positive for CD68 and HAM $[9,10]$. CD68 and S100 markers were positive in the specimen of our patient. Extracutaneous involvement is rare; eye, spleen, testicles, penis, pericardium, gastrointestinal system and kidneys may be involved. A patient with nonLCH should be evaluated for extracutaneous involvement. Radiological, biochemical and hematological investigations must be done to exclude xanthoma disseminatum, generalized eruptive histiocytoma and pancytopenia [2]. Our patient did not have any extracutaneous lesions and pancytopenia. No pathological findings were detected in her cranial and abdominal ultrasonograpy scans. Treatment for extracutaneous involvement may be required to decrease the mass effect of the lesions. Advanced treatment regimens and surgical approach may be considered in non-LCH, when there is major systemic involvement along with cutaneous involvement [11]. Only in the presence of cutaneous involvement, it does not require any treatment, as the lesions resolve spontaneously. The lesions in our patient disappeared without any treatment.

The non-LCH can mimic a number of neonatal skin lesions; including neonatal pustular melanosis, perinatal herpes simplex and Listeria monocytogenes, congenital candidiasis, neonatal varicella, syphilis, erythema toxicum, incontinentia pigmenti, neonatal disseminated hemangiomatosis, erythropoiesis dermica, and congenital leukemia [12]. Gram stain, potassium hydroxide 
$(\mathrm{KOH})$ and Tzanck preparations, and bacterial, viral, and fungal cultures help to differentiate the non-LCH from other disorders. TORCH serologies help to rule out congenital intrauterine infections. A skin biopsy specimen is, however, necessary for confirmation of the diagnosis.

The differential diagnosis of the disseminated histiocytoses is sometimes difficult because they occur with overlapping clinical and pathological findings. Nevertheless, the histiocytoses must be clearly differentiated from one another because they require different forms of treatment approaches and have different outcomes [9,10]. Although, the disease shows spontaneous involution, careful evaluation for systemic disease and longterm follow-up to detect relapse are essential in the management of these patients.

We would like to emphasize that non- $\mathrm{LCH}$ which predominantly affects the skin is benign and disappears without any treatment. A skin biopsy specimen is, however, necessary for confirmation of the diagnosis.

\section{REFERENCES}

[1] Minkov M, Prosch H, Steiner M, et al. Langerhans cell histiocytosis in neonates. Pediatr Blood Cancer 2005;45:8027. doi: $10.1002 /$ pbc. 20362

[2] Hart Isaacs, Jr. Fetal and neonatal histiocytoses. Pediatr Blood Cancer 2006;47:123-129. doi: 10.1002/pbc.20725

[3] Classen CF, Minkov M, Lehrnbecher T. The non Langerhans cell histiocytoses (Rare histiocytoses) - Clinical aspects and therapeutic approaches. Klin Pediatr 2016; 228(6-07):294-306. doi: 10.1055/s0042-109713.

[4] Walia M, Paul P, Mishra S, Mehta R. Congenital Langerhans cell histiocytosis: The self-healing variety. J Pediatr Hematol
Oncol 2004;26:398-402. doi: 10.1097/00043.426.20040600000014

[5] Haroche J, Abla O. Uncommon histiocytic disorders: RosaiDorfman, juvenile xanthogranuloma, and Erdheim-Chester disease. Hematology. Am Soc Hematol Educ Program 2015; 2015:571-8. doi: 10.1182/ asheducation-2015.1.571.

[6] Divaris DX, Ling FC, Prentice RS. Congenital self-healing histiocytosis. Report of two cases with histochemical and ultrastructural studies. Am J Dermatopathol 1991;13:481-7.

[7] Stein SL, Paller AS, Haut PR, et al. Langerhans cell histiocytosis presenting in the neonatal period: A retrospective case series. Arch Pediatr Adolesc Med 2001;155:778-83. doi:10.1001/ archpedi.155.7.778

[8] Rivera-Luna R, Alter-Molchadsky N, Cardenas-Cardos R, et al. Langerhans cell histiocytosis in children under 2 years of age. Med Pediatr Oncol 1996;26:334-43. doi:10.1002/ (SICI) 1096-911X(199605)26:5<334::AID-MPO6>3.0.CO;2-J

[9] Huang F, Arceci R. The histiocytoses of infancy. Semin Perinatol 1999;23:319-31. doi: 10.1016/s0146-0005(99)80040-8

[10] Weitzman S, Jaffe R. Uncommon histiocytic disorders: The non-Langerhans cell histiocytoses. Pediatr Blood Cancer 2005;44:1-9. doi: 10.1002/pbc.20246

[11] Mahajan VK, Sharma AL, Chauhan PS, Mehta KS, Sharma V, Sharma S. Xanthoma disseminatum: a red herring xanthomatosis. Indian J Dermatol Venereol Leprol 2013;79:253-4. doi: 10.4103/0378-6323.107655. PMID: 23442474 .

[12] Arico M, Danesino C, Pende D, et al. Pathogenesis of haemophagocytic lymphohistiocytosis. $\mathrm{Br} \mathrm{J}$ Haematol 2001;114:761-769. doi: 10.1046/j.1365-2141.2001.02936.x 